\title{
The application of Compressed Sensing Techniques in Radio Astronomy
}

\author{
Anna M. M. Scaife ${ }^{1}$ and Yves Wiaux ${ }^{2,3,4}$ \\ ${ }^{1}$ Dublin Institute for Advanced Studies (DIAS), 31 Fitzwilliam Place, Dublin 2, Ireland \\ email: ascaife@cp.dias.ie \\ ${ }^{2}$ Institute of Electrical Engineering, Ecole Polytechnique Fédérale de Lausanne (EPFL), CH-1015 \\ Lausanne, Switzerland \\ ${ }^{3}$ Institute of Bioengineering, Ecole Polytechnique Fédérale de Lausanne (EPFL), CH-1015, Lausanne, \\ Switzerland \\ ${ }^{4}$ Department of Radiology and Medical Informatics, University of Geneva (UniGE), CH-1211, Geneva, \\ Switzerland \\ email: yves.wiaux@epfl.ch
}

\begin{abstract}
Radio interferometry probes astrophysical signals through incomplete and noisy Fourier measurements. The optimal reconstruction of these signals is an important topic not only for current astronomical imaging but also that of the next generation of radio telescopes, for many of which image dynamic range is a key driver. The theory of compressed sensing demonstrates that incompletely sampled signals, such as those from an interferometer, may be accurately reconstructed when they are sparse or compressible in some basis. The introduction of an explicit sparsity constraint makes the method extremely versatile as it allows prior information on the signal to be introduced. Compressed sensing has been demonstrated to offer significant improvement over standard algorithms, and the flexibility of the framework and its implications for widefield imaging are compelling, as is its potential for influencing data acquisition methods and improving data storage and transport.
\end{abstract}

\section{Introduction}

Observations of the sky using interferometers are inherently under-sampled in angular frequency. This under-sampling is a result of the way in which these telescopes work and contrary to being a problem for astronomers is often used to advantage when extracting information on particular angular scales. However, for imaging applications the under-sampling inherent to interferometry can limit the fidelity of the reconstructed images as well as their dynamic range, and consequently significant effort and expertise is invested in optimal image reconstruction techniques. Compressed sensing represents a new approach to what is essentially a deconvolution problem in radio astronomy by providing a robust mathematical basis for accurate image reconstruction from sparsely sampled sky intensity distributions. In addition to its primary application at the image reconstruction level, compressed sensing also has implications at the acquisition level for the reduction in size of visibility datasets, by providing a mechanism for determining optimal observing practices for specific science cases, as well as at the post-reconstruction level for image datasets by returning a small number of basis coefficients which fully determine the image content. Such considerations for imaging and data storage are both increasingly important for the next generation of radio telescopes.

\section{Compressed Sensing}

The theory of compressed sensing, or compressive sampling, [1, 2, 3] states that signals which are sparse in some basis may be perfectly reconstructed when under-sampled at more than Nyquist rate, subject to a specific constraint. In mathematical terms, if we have a true signal distribution $x$, which, when sampled at Nyquist rate or better to give a set of real data $x=\left\{x_{i}\right\}_{1 \leq i \leq N}$, can be considered sparse in some basis, identified by the matrix $\Psi$, such that

$$
x=\Psi \alpha,
$$


where $\alpha$ represents the set of coefficients of basis $\Psi$ and is sparse such that it contains only a small number of non-negligible elements, $K \ll N$. Then if that signal is sampled by a set of noisy measurements $y$ in some other real sensing basis, identified by the matrix $\Phi$, such that

$$
y=\mathrm{M} \Phi \Psi \alpha+n,
$$

where $n$ is the noise and M represents a mask corresponding to the under-sampling in $\Phi$. The original signal can be accurately reconstructed as long as the measurement matrix $\Theta=\Phi \Psi$ satisfies an explicit restricted isometry property, or $R I P$.

A measurement matrix $\Theta$ can be said to satisfy this RIP if some constant $\delta_{K}$ exists such that

$$
\left(1-\delta_{K}\right)\left\|\alpha_{K}\right\|_{2}^{2} \leq\left\|\Psi \alpha_{K}\right\|_{2}^{2} \leq\left(1+\delta_{K}\right)\left\|\alpha_{K}\right\|_{2}^{2}
$$

for all vectors $\alpha_{K}$ with $\mathrm{K}$ non-zero coefficients.

If the corresponding $\Theta$ satisfies a RIP with $\delta_{2 K}<\sqrt{2}-1$ [4], then compressed sensing says that $x^{*}$ provides an accurate reconstruction of the original signal $x$. Importantly, many random sampling constructions respect the RIP with very high probability. For instance, the sensing obtained by a small number of randomly sampled measurements from a basis incoherent with the sparsity basis, in the sense that the vectors of the sparsity basis are not sparsely represented in the sensing basis, will almost always ensure this property when the number of measurements is bigger than only a few multiples of the image sparsity level.

Consequently, compressed sensing reconstruction depends on finding the vector $\alpha_{K}$ which is sparse. In practice the signal reconstruction from the measurement vector $y$ can be achieved using a Basis Pursuit method including a constraint on the $\ell_{1}$ norm of $\alpha$. The expression that is solved is then

$$
\min \left\|\alpha^{\prime}\right\|_{1} \text { subject to }\left\|y-\mathrm{M} \Theta \alpha^{\prime}\right\|_{2} \leq \epsilon
$$

The $\ell_{1}$ norm is simply the sum of the magnitudes of the co-efficient $\alpha$, i.e. $\|\alpha\|_{1}=\sum_{i}\left|\alpha_{i}\right|$. This constraint can be considered as a logarithmic Laplacian prior on the values of $\left|\alpha_{i}\right|$. The Laplacian function is sharply peaked with wide tails compared to, say, the Gaussian function, which is broad and falls away quickly and would be represented by the $\ell_{2}$ norm. The effect of this sharply peaked function is to explicitly assume sparsity for the coefficients $\alpha$.

The term $\epsilon$ is due to the noise and, for Gaussian noise, may be considered as the percentile of a standard chi-square distribution where $p\left(\chi^{2} \leq \epsilon^{2}\right)=\eta$. As with standard maximum likelihood and chi-square the choice of $\epsilon$ is crucial, with too large a value leading to a poor constraint and too small a value leading to over-fitting. In the case that the solution to this problem is $\alpha^{*}$, then the corresponding signal reconstruction is simply $x^{*}=\Psi \alpha^{*}$, where $\alpha^{*}$ has $K$ elements.

In the case of noiseless data it has been shown that the original signal $x$ can be recovered exactly from undersampled measurements [4], i.e. $x=x^{*}$. However interferometric visibilities will never be entirely noiseless because of the hardware required to measure them. In the case of noisy measurements there is still strong stability and the accuracy of the result, characterised by $\left\|\alpha-\alpha^{*}\right\|_{2}$, has been shown to be bounded by limits defined by both the noise (proportional to $\epsilon$ ) and also the degree of non-exact sparsity of the signal in the basis $\Psi$.

\section{Compressed sensing for radio interferometry}

In radio astronomy we may consider our original sky signal multiplied by the aperture illumination function of our telescope as $x$. When we observe with an interferometer we sample that signal using a noisy Fourier basis, $\Phi$, such that

$$
y=\mathrm{M} \Phi x+n
$$


The sampling is incomplete in Fourier space due to the finite number of baselines in our interferometer and this is included through the matrix M. The sampled measurements, $y_{i}$, are simply the recovered visibilities and $n_{i}$ is the noise on a measured visibility and is Gaussian. If our original sky signal $x$ can be represented in some sparse basis $\Psi$ then we can apply the principles of compressed sensing to its reconstruction as described in $\S 2[10]$ and recover $x^{*}$ from $y$.

One potential limitation of the method for radio interferometry is that compressed sensing is optimally performed using a uniformly random distribution of measurements, in this case a random sampling of Fourier frequencies. Since the sampling distribution produced by interferometers is deterministic in Fourier space this is non-optimal. However this problem may be tackled by re-weighting the visibility distribution in the same way as is done to improve the form of the synthesised beam using so-called normal weighting, which homogenises the spatial weight of the distribution of visibilities in $u v$ space subject to a loss in sensitivity commensurate with the degree of re-weighting. For future applications it is also conceivable that observing practices may be implemented that randomise to some degree the visibility distribution. However, neglecting any modifications to the observing process it has already been shown that some deterministic sampling distributions are in fact suitable for compressed sensing [5].

\section{Applications and Prospects}

Compressed sensing is optimised for signals which are readily expressible in a sparse basis and are undersampled when measured. A good example of this situation is given by a sky signal composed solely of point-like objects. Such sources may be readily expressed by a minimum number of coefficients using a Dirac basis (i.e. $\delta$-functions). Consequently a field of point sources measured by a limited number of visibilities is an instantly recognisable application for compressed sensing, notably because the Dirac signal basis and Fourier sampling basis are maximally incoherent and therefore easily satisfy the RIP. Such a situation is also optimal for the widely used CLEAN deconvolution algorithm, to which compressed sensing bears a pleasing similarity. Indeed in the case of a Dirac basis the two methods are highly comparable [10], differing only by compressed sensing explicitly enforcing sparsity through the $\ell_{1}$ norm term in Eq. 4 .

A major advantage of the compressed sensing framework is its flexibility for including additional prior information on the sky signal. A good example of this is the application of compressed sensing to reconstructing cosmic string signals [8]. String signals exhibit sparse gradient magnitudes due to the Kaiser-Stebbins effect. The natural sparsity of the signals in this respect makes the versatile framework of compressed sensing ideal for reconstruction of their spatial signature from under-sampled data. In this case the compressed sensing minimisation problem is adapted such that it is not the $\ell_{1}$ norm of the basis coefficients that is minimised subject to a chi-square constraint, but instead the Total Variance (TV) norm of the candidate reconstruction. The TV norm of a signal is simply the $\ell_{1}$ norm of its gradient, in this case enforcing the sparsity known to result from the Kaiser-Stebbins effect for string signals.

A recent enhancement of image reconstruction for radio astronomy using compressed sensing [6] has come from its combination with spread spectrum techniques [9, 7]. This combination has applications for interferometers with wide fields of view, such as the LOFAR and ASKAP arrays. Wide fields of view are problematic in imaging for a number of reasons. The small fields of view observed by most operating telescopes have generally enabled the sky to be imaged using a planar approximation, but the new generation of radio telescopes with wide fields of view will no longer be able to make this simplification. In addition, the loss of co-planarity of the interferometer array elements will also cause image distortions by introducing non-zero terms in the radial direction cosine, $w$. However, non-zero terms in $w$ have been shown to produce a spread spectrum phenomenon, enhancing visibility acquisition from the perspective of image reconstruction, by leading to optimal incoherence of the measurement basis, irrespective of sparsity basis [9]. Consequently compressed sensing techniques have begun to provide an elegant solution to this problem and details of the intrinsic advantages of the $w$ component and spherical imaging for wide-field interferometry with compressed sensing are reviewed in a separate article of the current proceedings [11]. 


\section{Conclusions}

Compressed sensing offers a new framework for image reconstruction in radio interferometry. It allows a solution to the inverse problem of image reconstruction from incomplete and noisy Fourier measurements to be found when regularized by the definition of global minimization problems in which a generic sparsity or compressibility prior is explicitly imposed. It has been demonstrated as a useful technique for both currently operating telescopes and the next-generation of radio telescopes, and has the potential to solve a number of the image reconstruction issues currently limiting both the fidelity and dynamic range of images from these facilities. These considerations are crucial for next generation instruments such as the SKA and LOFAR telescopes, where dynamic range is a key goal for their technical development. In addition it has a number of currently unexplored applications in optimal data gathering and storage, where it is becoming evident that a lack of sparse or compressible representations for telescope measurements is increasingly problematic from both a data storage and a data transport viewpoint. Compressed sensing allows a reconstructed signal to be represented completely through a sparse set of $K$ significant basis coefficients only and therefore may provide at least a partial practical solution to these problems.

\section{References}

[1] E. J. Candès, J. Romberg and T. Tao, "Robust uncertainty principles: exact signal reconstruction from highly incomplete frequency information", IEEE Trans. Inform. Theory, 52, 489, 2004

[2] E. J. Candès, J. Romberg and T. Tao, "Stable signal recovery from incomplete and inaccurate measurements", Comm. Pure and Appl. Math., 59, 1207, 2005

[3] E. J. Candès, "Compressive sampling", Proc. Int. Congress Math. Vol. 3. Euro. Math. Soc., p. 1433, 2006

[4] E. J. Candès, "The restricted isometry property and its implications for compressed sensing", Compte Rendus de l'Academie des Sciences, Paris, Series I, 346, 589, 2008

[5] B. Matei and Y. Meyer, "A variant of compressed sensing", Rev. Mat. Iberoamericana, 25, 2, 669-692, 2009

[6] J. D. McEwen and Y. Wiaux, "Compressed sensing for wide-field radio interferometric imaging", arXiv, arXiv:1010.3658, 2010

[7] G. Puy, P. Vandergheynst, R. Gribonval and Y. Wiaux, "Universal and Efficient Compressed Sensing Strategy through Spread Spectrum Modulation", submitted to IEEE Trans. Signal Process., preprint EPFL infoscience 163850, 2011

[8] Y. Wiaux, G. Puy and P. Vandergheynst, "Compressed sensing reconstruction of a string signal from interferometric observations of the cosmic microwave background", MNRAS, 402, 2626, 2010

[9] Y. Wiaux, G. Puy, Y. Boursier and P. Vandergheynst, "Spread spectrum for imaging techniques in radio interferometry", MNRAS, 400, 1029, 2009

[10] Y. Wiaux, L. Jacques, G. Puy, A. M. M. Scaife and P. Vandergheynst, "Compressed sensing imaging techniques for radio interferometry", MNRAS, 395, 1733, 2009

[11] J. D. McEwen and Y. Wiaux, "Intrinsic advantages of the w component and spherical imaging for wide-field radio interferometry", URSI General Assembly and Scientific Symposium, 2011 